\title{
Compressão traqueal como método auxiliar no diagnóstico radiológico do colapso de traquéia cervical
}

Júlio Carlos CANOLA ${ }^{1}$ Naida Cristina BORGES ${ }^{1}$

Correspondência para: JÚLIO CARLOS CANOLA

Departamento de Clínica e Cirurgia

Veterinárias

Faculdade de Ciências Agrárias e

Veterinárias da UNESP

Campus de Jaboticabal

Via de Acesso Prof. Paulo Donato

Castellane, s/n

14884-900 - Jaboticabal - SP

canola@fcav.unesp.br

naidacb@ig.com.br

Recebido para publicação: 17/07/2003 Aprovado para publicação: 13/07/2005

1 - Departamento de Clínica e Cirurgia da Faculdade de Ciências Agrárias e Veterinárias da Universidade Estadual Paulista, Campus de Jaboticabal, Jaboticabal - SP

\section{Resumo}

Palavras-chave:

Colapso.

Traquéia.

O colapso de traquéia é uma afecção freqüente em cães idosos e principalmente de raças pequenas, caracterizada por flacidez e perda de sustentação dos anéis traqueais com conseqüente diminuição do espaço luminal. O diagnóstico baseia-se nos sinais clínicos e nos exames de raios-x, fluoroscopia, ultra-sonografia e traqueobroncoscopia. Quarenta cães adultos de pequeno porte e de diferentes raças, vinte hígidos e vinte com suspeita dessa afecção, tiveram as regiões cervical e torácica radiografadas em projeções laterais com e sem compressão ventral da traquéia. A leve pressão exercida sobre a traquéia cervical foi realizada na entrada do tórax, com o auxílio de uma "pêra de borracha" adaptada a uma haste de vidro. Independente da aplicação ou não da compressão, os vinte animais sem anormalidades das vias respiratórias tiveram mudanças sutis no diâmetro traqueal. Os cães com suspeita clínica de colapso de traquéia apresentaram ou não estreitamento deste órgão, dependendo da fase respiratória em que se encontravam durante a projeção radiográfica simples, não permitindo, muitas vezes, o diagnóstico definitivo. Todavia, todos os animais deste grupo, quando submetidos à técnica de compressão da traquéia apresentaram, ao raio-x, estreitamento local deste órgão, caracterizando o colapso de traquéia. $O$ método mostrou-se eficiente, prático e seguro no diagnóstico do colapso de traquéia da região cervical, eliminando a necessidade de exposições radiográficas em diferentes momentos do ciclo respiratório, sem custos adicionais.

\section{Introdução}

O colapso de traquéia é causa comum de obstrução das vias aéreas superiores em cães. O primeiro caso foi relatado em 1941 e até 1970 poucas informações foram adicionadas para o adequado entendimento desta afecção ${ }^{1}$.

Esta enfermidade é uma forma de obstrução da traquéia causado por flacidez e achatamento dos anéis cartilagionosos ${ }^{2}$. É também referida como um síndrome que atinge cães de raças pequenas que, ocasionalmente, pode ocorrer em raças grandes ou em outras espécies animais ${ }^{3}$. O problema é comumente observado em cães de meia idade ou idosos, em geral entre os seis a sete anos de vida ${ }^{4,5}$. Entretanto, têm sido diagnosticados casos em cães com idade entre um e cinco anos portadores de problemas respiratórios ${ }^{2}$.

A etiologia da afecção é obscura e provavelmente multifatorial, mas macroscópica e histologicamente ocorrem alterações degenerativas nos anéis cartilaginosos e no ligamento traqueal dorsal, a cartilagem tornase hipocelular com perda da rigidez e habilidade de manter-se em sua conformação normal durante o ciclo respiratório, com conseqüente achatamento dorsoventral da traquéia e redução do lúmen traqueal no sentido transversal ${ }^{2,5,6,7,8}$. Os sinais clínicos típicos são tosse crônica paroxística, denominada "tosse 
de ganso", mímica de vômito e dispnéia ${ }^{1,4,7}$.

O diagnóstico presuntivo do colapso de traquéia baseia-se no histórico, sinais e sintomas clínicos, porém o diagnóstico definitivo só pode ser obtido por meio de radiografias, fluoroscopia, ultra-sonografia e traqueobroncoscopia.

Radiografias torácicas são eficazes em detectar colapso de vias aéreas em $59 \%{ }^{9}$ a $84 \%{ }^{8}$ dos casos, entretanto, a musculatura cervical e o esôfago freqüentemente sobrepõem a traquéia dificultando o claro delineamento traqueal na região cervical e na entrada do tórax ${ }^{10,11}$. Projeções radiográficas laterais durante as fases inspiratória e expiratória são indicadas para demonstrar colapso traqueal do segmento cervical e torácico, respectivamente, além de permitir o diagnóstico de doenças cardíacas e pulmonares concomitantes ${ }^{1,5,7}$.

Em muitos cães, a radiografia estática é insuficiente para diagnosticar o colapso das vias aéreas, particularmente, quando a porção intratorácica está envolvida. Nestes casos, a fluoroscopia é freqüentemente indicada, pois permite uma avaliação dinâmica da traquéia em todas as fases da respiração $0^{1,2,5}$. Neste sentido, a ultrasonografia também é o método indicado para avaliar os movimentos traqueais. Neste exame, a traquéia normal aparece hipercóica e com conformação circular ao corte transversal, enquanto a traquéia colapsada é vista em formato oval ou em forma de feijão, quando o pescoço do animal é mantido hiperestendido ou em posição neutra ${ }^{10}$.

A traqueobroncoscopia permite uma avaliação precisa da conformação do lúmen traqueal e dos graus de colabamento da traquéia, variando do grau I com redução de $25 \%$ do diâmetro do lúmen traqueal, ao grau IV, cujo lúmen está obliterado e o músculo traqueal em contato com a cartilagem $^{12}$. Esta técnica porém, que requer anestesia geral, agrava os sintomas de tosse e provoca descompensação em cães obesos em função do depressão respiratória induzida combinada com o aumento do trabalho inspiratório ${ }^{7}$.
O colapso de traquéia é uma doença de diagnóstico relativamente fácil e que permite a utilização e combinação de várias técnicas de imagem para seu reconhecimento. No entanto, cada método apresenta algum inconveniente ou desvantagem de aplicação, necessitando ainda de estudos para que sejam aprimorados em vários aspectos. Este trabalho propõe um método auxiliar no diagnóstico radiológico desta síndrome, de fácil realização, custo adicional baixo e que permite dirimir dúvidas que são geradas pela sobreposição de tecidos moles sobre a traquéia na região cervical e entrada do tórax.

\section{Materiais e Métodos}

Vinte cães adultos de diferentes raças e porte pequeno, sem anormalidades respiratórias tiveram as regiões cervical e torácica radiografadas em projeção lateral com o pescoço em posição normal. Em seguida, com o animal na mesma posição, uma segunda exposição foi realizada. Todavia, desta vez, uma "pêra de borracha", adaptada a uma haste de vidro de aproximadamente $25 \mathrm{~cm}$ foi posicionada na entrada do tórax e a região ventral da traquéia levemente pressionada durante a exposição radiográfica

Após avaliação clínica, outros 20 animais com suspeita de colapso de traquéia foram encaminhados para a avaliação radiográfica e submetidos ao mesmo procedimento radiográfico descrito anteriormente.

As projeções radiográficas, além de permitirem comparar morfologicamente a imagem da traquéia antes e após a compressão, serviram para avaliar a silhueta cardíaca, a cavidade torácica e os diferentes padrões pulmonar.

\section{Resultados e Discussão}

A traquéia foi facilmente identificada radiograficamente em todos os 40 cães examinados. Nos 20 animais sem anormalidades aparentes das vias respiratórias, constatou-se

Braz J vet Resanim Sci, São Paulo, v. 42, n. 6, p. 414-418, 2005 


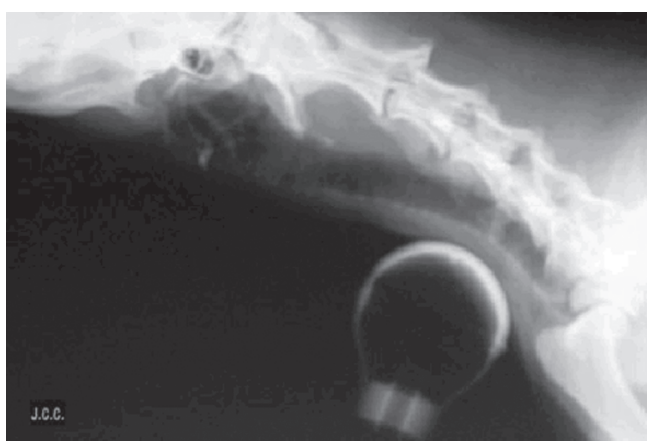

Figura 1 - Projeção radiográfica lateral direita da região cervical de um cão da raça Poodle, idade 10 anos, sem colapso de traquéia Observarque mesmo comaaplicação datécnica compressiva coma "pêra de borracha" não ocorreu mudança no diâmetro luminal da traquéia

mudanças sutis no diâmetro traqueal sem ou com a aplicação da compressão na entrada do tórax no momento do exame radiográfico. Achados semelhantes foram descritos por outros autores ${ }^{13}$ que afirmam haver pequenas alterações no diâmetro traqueal em função da respiração e às vezes tosse em animais hígidos no momento do exame radiológico. Acrescentam, ainda, que é dificil estabelecer uma clara distinção entre um estreitamento normal da traquéia e uma situação de colapso, porém se ocorre uma diminuição do diâmetro do lúmen maior que $50 \%$, o colapso deve ser considerado. Neste grupo de animais, mesmo com a aplicação de pressão não verificou-se redução significativa no diâmetro traqueal (Figura 1).

grupo de animais com suspeita de

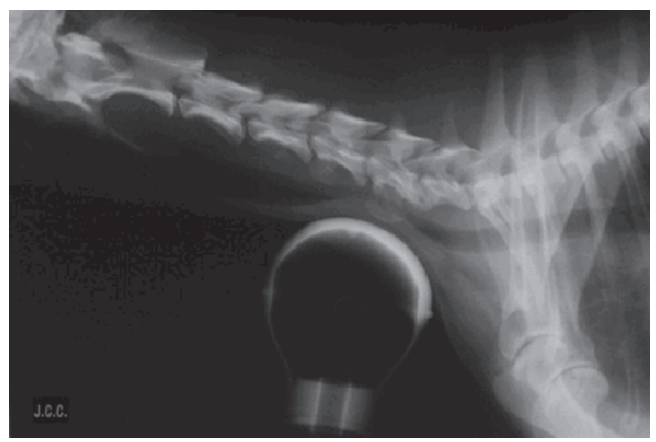

Figura 3- Projeção radiográfica lateral direita da região cervical do cão daFigura 2, submetido ao método de compressão Observar a redução total do lúmen traqueal, decorrente da flacidez dos anéis cartilaginosos, no local da pressão exercida com auxílio da pêra de borracha, confirmando a suspeita radiográfica de colapso de traquéia

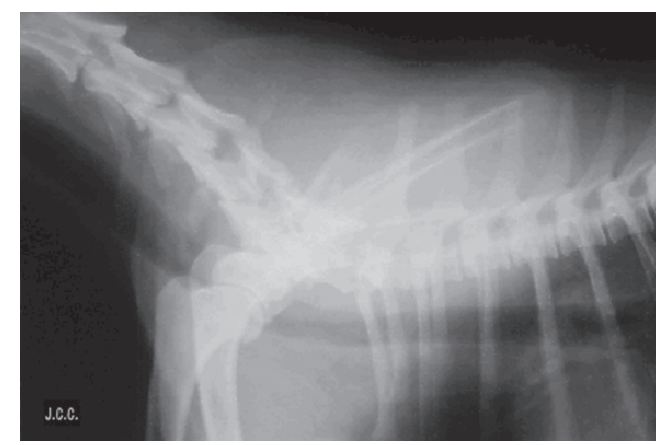

Figura 2 - Projeção radiográfica lateral direita da região cervical de um cão da raça Poodle, idade 9 anos, com suspeita clínica de colapso detraquéia. Notarna regiãocenvical a sobreposição do músculotraqueal sobre o dorso datraquéia, durante a inspiração, indicativo de colapso de traquéia

colapso de traquéia revelou, com certa freqüência, ao exame radiológico sem o método auxiliar de compressão, leve estreitamento deste órgão não permitindo o diagnóstico definitivo da afecção (Figura 2). Tais achados são concordantes com as citações de Burk e Ackermann ${ }^{13}$; Ettinger e Feldman $^{5}$; Fossum ${ }^{2}$ e Jerram e Fossum ${ }^{1}$ a cerca do fato desta síndrome ser diagnosticada em exame radiográfico simples, quando há estreitamento da traquéia durante a respiração normal ou forçada, onde o estreitamento cervical caudal é mais visível na inspiração e o traqueal intratorácico na expiração. Concordam, também, com Rudorf, Herrtage e White ${ }^{10}$, em relação aos aspectos que podem interferir com os resultados do exame radiológico, quais sejam, a dificuldade em obter uma radiografia no momento exato da inspiração ou expiração, o posicionamento inadequado do animal e a superposição de tecidos moles na região cervical $^{11}$

Este mesmo grupo de animais, ao ser submetido ao exame radiográfico compressivo apresentou diagnóstico positivo para o colapso de traquéia (Figura 3). O princípio desta técnica baseia-se no fato de que animais com esta síndrome apresentam anéis cartilaginosos flácidos e não resistentes a qualquer tipo de pressão. Nestes casos, os anéis cartilaginosos possuem menor quantidade de glicosaminoglicanos e água, a matriz cartilaginosa apresenta poros, fissuras e pequena quantidade 
de condrócitos, as fibras cartilaginosas são menores e variam enormemente em diâmetro. Estas mudanças estruturais promovem enfraquecimento dos anéis que passam a não suportar adequadamente pressões ${ }^{14,15,16}$.

Radiografias simples do tórax são eficazes em detectar colapso de vias aéreas em $59 \%$ dos casos ${ }^{9}$. Acredita-se que, com a técnica compressiva proposta nesse trabalho, esse percentual possa alcançar 100\% de precisão, pois quando comparada a uma radiografia convencional permite a minimização de erros grosseiros relativos a posicionamentos inadequados e superposição de tecidos moles localizados na região cervical.

Em relação a fluoroscopia, traqueobroncoscopia e ultra-sonografia, o método compressivo não representa risco para o animal. Johnson ${ }^{7}$ lembra que a traqueobroncoscopia requer anestesia geral, agrava os sintomas de tosse e provoca descompensação em cães obesos. Além disso, ao serem considerados outros aspectos como custo e complexidade de execução, essas técnicas de imagem são mais onerosas que o método relatado neste trabalho e requerem pessoal qualificado para executá-las. Burk e Ackerman $^{13}$ citam vários estudos usando a administração intratraqueal de bário ou contraste iodado solúvel em água, os quais nada acrescentam, quando comparados aos resultados obtidos com radiografias simples cuidadosamente posicionada e com exposição apropriada no diagnóstico do colapso de traquéia.

Alguns cuidados devem ser tomados no momento da aplicação da técnica doravante proposta sob o risco de não se obter a qualidade esperada no exame. Verificou-se que pressões muito leves podem não promover o colabamento desejado, dependendo da flacidez da traquéia em estudo. $O$ posicionamento incorreto da "pêra de borracha" pode provocar a sobreposição da sua extremidade distal com a luz da traquéia, colabada ou não, levando a resultado falso negativo. Neste caso, a técnica deverá ser repetida.

\section{Conclusões}

método auxiliar de diagnóstico radiográfico, proposto neste trabalho, utilizando-se de uma "pêra de borracha" posicionada corretamente na entrada do tórax e promovendo uma leve pressão na traquéia, mostrou-se eficiente, prático e seguro no diagnóstico do colapso de traquéia. Sua aplicação permite detectar casos de colapso traqueal de grau inferior a $25 \%$ que não são confirmados em raio-x convencional e sanar dúvidas deixadas pela sobreposição dos tecidos moles no momento do exame. Elimina a necessidade de radiografias em diferentes momentos do ciclo respiratório no diagnóstico de colapso de traquéia. Enfim, revelou-se extremamente simples e preciso, além de não representar custos adicionais.

\section{Tracheal compression as auxiliary method in the radiologic diagnosis of the cervical tracheal collapse}

\section{Abstract}

Tracheal collapse is generally a syndrome of small-breed dogs that is occasionally reported in larger breeds or other species. It is characterized by weakening and loss of adequately support dynamic intraluminal pressure changes. The diagnosis bases on the clinical signs and in the exams of ray-x, fluoroscopy, ultrasonography and tracheobronchoscopy. Forty adult small dogs of different breeds, twenty health and twenty with suspicion of that tracheal collapse. They had the cervical and thoracic areas $\mathrm{x}$-rayed in lateral projections with and without ventral compression of the trachea. The light pressure
Key-words:

Collapse.

Trachea radiography.

Dog. 
in the cervical trachea was accomplished in the thoracic inlet with a "eraser pear" adapted in a glass stem during the radiographic examination. The twenty animals without abnormalities of the airways presented subtle changes in the diameter traqueal independent or not of the compression. The suspicious dogs of collapse presented or no narrowing of this organ depending on the breathing phase in that they were during the radiographic projection not allowing definitive diagnosis. However when all animals of this group were submitted to the technique of compression of the trachea they presented local narrowing of this organ characterizing tracheal collapse. The method was shown efficient, practical and safe in the diagnosis of cervical tracheal collapse. Besides to eliminate the need of x-rays in different moments of the breathing cycle and not to represent additional costs.

\section{Referências}

1 JERRAM, R. M.; FOSSUM, T. W. Tracheal collapse in dogs. Compendium on Continuing Education for the Practicing Veterinarian, v. 19, n. 9, p. 1049-1060, 1997

2 FOSSUM, T. W. Small animal surgery. 2. ed. St. Louis: Mosby, 2002. p. 740-744.

3 RADLINSKY, M. G.; FOSSUM, T. W. Tracheal collapse in a young boxer. Journal of the American Animal Hospital Association, v. 36, n. 4, p. 313-316, 2000.

4 BOJRAB, M. J. Disease mechanisms in small animal surgery. 2. ed. Philadelphia: Lea \& Febiger, 1993. 1271

5 ETTINGER, S. J.; FELDMAN, E. C. Textbook of veterinary internal medicine. 4. ed. São Paulo: Manole, 1995. v. 1, 1081 p.

6 DUNN, J. K. Tratado de medicina de pequenos animais. São Paulo: Roca, 2001. 1075 p.

7 JOHNSON, L. Tracheal collapse. Diagnosis and medical and surgical treatment. Veterinary Clinics of North America:Small Animal Practice, v. 30, n. 6, p. 1253-1267, 2000

8 WHITE, R. A. S.; WHILLIANS, J. M. Tracheal collapse is there really a role for surgery? A survey of 100 cases. Journal of Small Animal Practice, v. 35, p. 191-196, 1994

9 TANGNER, C. H.; HOBSON, H. P. A retrospective study of 20 surgically managed cases of collapsed trachea. Veterinary Surgery, v. 11, n. 4 , p. 146-149, 1982.

10 RUDORF, H.; HERRTAGE, M. E.; WHITE, R. A. S Use of ultrasonography in the diagnosis of tracheal collapse. Journal of Small Animal Practice, v. 38, n. 11 , p. $513-518,1997$.

11 THRALL, D. E. Textbook of veterinary diagnostic radiology. 3. ed. Philadelphia: Saunders, 1998. 663 p.
12 HOOSGOOD, G. Sistema respiratório. In: HARARI, J. Cirurgia de pequenos animais. Porto Alegre: Artmed, 1999. p. 103-105.

13 BURK, R. L.; ACKERMAN, N. Small animal radiology and ultrasonography. A diagnostic atlas and text. 2. ed. Philadelphia: Saunders, 1996. 644 p.

14 DALLMAN, M. J.; MCCLURE, R. C.; BROWN, E. $M$. Histochemical study of normal and collapsed tracheas in dogs. American Journal of Veterinary Research, v. 49, n. 12, p. 2117-2125 1988.

15 DALLMAN, M. J.: MCCLURE, R. C.: BROWN, E. $M$. Normal and collapsed trachea in the dog: scanning electron microscopy study. American Journal of Veterinary Research, v. 49, n. 10, p. 2110-2116, 1985

16 HAMAIDE, A. et al. Effects of age and location on the biomechanical and biochemical properties of canine tracheal ring cartilage in dogs. America Journal of Veterinary Research, v. 59, n. 1, p. 18-22, 1998 